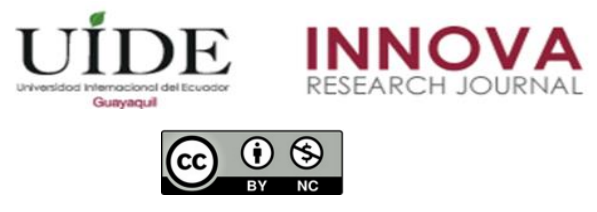

INNOVA Research Journal, ISSN 2477-9024

(Septiembre-Diciembre 2019). Vol. 4, No.3.2 pp. 172-187

DOI: https://doi.org/10.33890/innova.v4.n3.2.2019.1208

URL: http://revistas.uide.edu.ec/index.php/innova/index

Correo: innova@uide.edu.ec

\title{
Desempeño de las cien firmas más grandes y el crecimiento de la economía ecuatoriana. 2010-2016
}

\section{Performance of the one hundred largest firms and the growth of the Ecuadorian economy. 2010-2016}

María Augusta Játiva Pesantes

Jaime Leopoldo Cabezas Maldonado

Universidad Internacional del Ecuador, Ecuador

Autor por correspondencia: mjativa@uide.edu.ec; jacabezasma@uide.edu.ec

Fecha de recepción: 24 de septiembre del 2019 - Fecha de aceptación: 13 de diciembre del 2019

\section{Resumen}

El presente estudio compara el comportamiento de las cien mayores firmas privadas registradas ante la Superintendencia de Compañías que se corresponden con la evolución del ingreso, renta o Producto Interno Bruto -PIB- del Ecuador de los sectores económicos, durante el período comprendido entre 2010 y 2016; incluye el desempeño del empleo, la inversión y los precios, por ser datos significativos. El PIB es una magnitud que sirve para medir el comportamiento de una economía, su inversión y empleo; si su medición se muestra creciente, habrá mayor bienestar en la sociedad. El nivel de empleo se relaciona directamente con la forma como el talento humano contribuye a esa producción de manera plena, no precaria y mejora aún más si hay un mejor nivel de capitalización, por tanto, inversión. Por otro lado, toda firma es una organización que busca alcanzar los fines usando los medios y coordinando los intercambios tanto en el mercado de factores como en el de productos y opera en cumplimiento de la función empresarial para alcanzar objetivos valiosos para la sociedad.

Palabras clave: PIB; firma; crecimiento; empleo; función empresarial

\begin{abstract}
The present study compares behavior of the hundred largest private firms registered with the Superintendence of Companies that correspond to the evolution of income, income or Gross Domestic Product -GDP- of Ecuador by economic sectors, during the period between 2010 and 2016; It includes the performance of employment, investment and prices, as they are significant data. The GDP is a magnitude that serves to measure the behavior of an economy, its investment and employment; If your measurement shows increasing, there will be greater welfare in society. The level of employment is directly related to the way human talent contributes to this production in a full, non-precarious way and improves even more if there is a better level of capitalization, therefore, investment. On the other hand, every firm is an organization that seeks to achieve the ends using the media and coordinating exchanges in both the factor and product markets and operates in compliance with the corporate function to achieve valuable objectives for society.
\end{abstract}

Key words: GDP; firm; growth; employment; business function 


\section{Introducción}

Esta investigación contrasta el volumen y el crecimiento del Producto Interno Bruto PIB del Ecuador, total y por industrias o sectores económicos con el crecimiento del ingreso de las cien mayores firmas privadas registradas ante la Superintendencia de Compañías (Superintendencia de Compañías, 2018) y que se corresponden con esos sectores económicos, durante el período comprendido entre los años 2010 y 2016. Se presentan los resultados más significativos del crecimiento y participación de las cien firmas más grandes por ventas y el aporte que representan en varios sectores económicos de acuerdo a su posición en el ranking, es decir la clasificación de mayor a menor, útil para establecer algunos criterios de valoración.

Este estudio recopila la información relevante y económica de las cien mayores empresas ecuatorianas en un lapso de siete años desde el 2010 al 2016. Incluye el resultado del registro cronológico de su trayectoria en ranking por ingresos, número de empleados, activos e inflación.

Se busca establecer una comparación entre el ciclo macroeconómico y el comportamiento microeconómico de las empresas. Es decir, en base a la estrategia de las firmas: sus fines y los medios usados, el plan y su ejecución, estas firmas siguieron creciendo a pesar de las fluctuaciones de la economía en la mayoría de los casos, aunque algunas podrían mostrar un comportamiento que cedió al declive económico de la economía ecuatoriana.

Se consideran los resultados importantes de la función empresarial cumplidas por las empresas líderes de cada uno de los sectores de la economía, es decir como la competitividad de la propia empresa, su estructura, sus estrategias y su entorno, afectaron en la mayoría de los casos positivamente para seguir prosperando en ingresos, generación de empleo, crecimiento de sus niveles de inversión medido por medio del crecimiento real de sus activos y el entorno de los precios, los cuales afectaron positivamente a la mayoría de las firmas.

Fue posible comprobar que las cien firmas líderes de algunos sectores sobrevivieron a la fase recesiva del ciclo o eventualmente a excelentes planes para afrontar creativamente las dificultades que presentó el entorno.

\section{Metodología}

Este estudio demuestra que la empresarialidad es parte de la naturaleza de los seres humanos, buscando alcanzar sus fines, partiendo de situaciones poco satisfactorias para lograr finalmente sus metas en un ambiente de incertidumbre inerradicable unas veces y otras de un manejo controlado de los riesgos, siendo su resultado ganancias o pérdidas. (Mises, 2005)

También busca establecer el estado de nuestra economía durante los últimos siete años, para verificar el comportamiento de los agregados relevantes comparables con la evolución de los datos de estas cien firmas en cuanto a crecimiento del ingreso, empleo e inversión privada, desde la perspectiva de los indicadores macroeconómicos, enfatizando las ramas de actividad económicas implicadas. El plan original fue hacerlo desde el año 2007, es decir por un periodo de diez años, pero debido a la falta de información completa de las empresas, finalmente el estudio se centró en el período señalado de siete años. 
Describimos el comportamiento de las ventas o ingresos de estas empresas, comparando con el desempeño de la renta nacional o PIB. También el impacto en el pleno empleo por parte de estas empresas y los niveles de activos alcanzados, los cuales se contrastan con la formación bruta de capital fijo o inversión de todo el sector privado de la economía. En materia de precios solo se pudo mostrar la evolución de los precios sectoriales en base al deflactor del PIB.

El método usado para este estudio es uno del tipo empírico, histórico y deductivo con presentación de evidencia tanto del PIB calculado por el Banco Central del Ecuador (Banco Central del Ecuador, 2017) así como la información constante en el ranking preparado por la Superintendencia de Compañías de las diez mil firmas sujetas a su control, habiendo escogido las cien primeras del ranking y su correspondiente clasificación. Este ranking se refiere a aquellas firmas organizadas de conformidad con la Ley de Compañías del Ecuador, básicamente sociedades por acciones y compañías limitadas, las que presentan todos los años información económica y financiera de su desempeño ante este organismo de control.

Cualquier diferencia en los datos sería atribuible a la forma como ha sido compilada esta información, por ese organismo de control societario. Expresamente no se incluye a las firmas del sistema financiero, las que son supervisadas por otra institución de control del Estado, así como también a los monopolios del sector público, los que obedecen a dinámicas que no se ajustan a la visión de sociedades eficientes con fines de lucro.

Para el PIB industrial, se ha utilizado la información que procesa el Banco Central del Ecuador, desde hace muchos años y que se han adaptado para fines de este estudio y que consta en sus boletines estadísticos mensuales. (Banco Central del Ecuador, 2017).

Si bien se puede encontrar un sinnúmero de artículos académicos haciendo mención al desempeño de empresas, estos estudios no están organizados con la metodología de un ranking, sino más bien en estudios por sectores económicos, con criterios muy dispersos que enfatizan el desempeño de las medianas y pequeñas empresas, su grado de innovación o su aporte a la responsabilidad social, etc. Por lo dicho estas firmas no son parte del ranking de las cien mayores del Ecuador.

El presente trabajo tiene como objetivo mostrar por la vía de la comparación si las firmas representativas de la industria respectiva, es decir las más grandes de cada sector, rama de actividad o industria, contribuyeron en mayor o menor medida a la producción del país o ingreso de cada sector estudiado y al crecimiento del mismo. El PIB es una magnitud de flujo, no de stock como sería la riqueza acumulada por las familias y las firmas como personas jurídicas con fines de lucro y que en macroeconomía se contabiliza de acuerdo a la metodología del flujo circular, agregando solamente las ventas de los bienes y servicios finales producidos e intercambiados a precios de mercado durante la etapa de estudio. (Mankiw, 2012)

Por otro lado, las firmas han sido definidas así: en una versión estática, neoclásica y tayloreana: Empresa, reconocida como el espacio en donde se desarrolla un negocio, dedicado a cualquier actividad rentable, (Koontz, 2012). La empresa es el grupo de individuos u organizaciones que pretenden obtener una ganancia mediante la oferta de productos que 
satisfacen las necesidades de las personas, (Ferrel, 2010). Esfuerzo organizado de los individuos para producir y vender, con fines de lucro, bienes y servicios que satisfacen las necesidades de una sociedad. (Pride, 2017)

La escuela austriaco libertaria de economía, afirma que la función empresarial es ejercida por todos los individuos que actúan para modificar el presente y conseguir objetivos en el futuro, mediante una actitud emprendedora de darse cuenta de nuevos medios y fines, quienes unen y vinculan los conceptos de acción y empresa, acción humana que incluye decisiones optimizadoras esencialmente empresariales, en las que se incorporan planes, siempre enfrentadas al futuro bajo una incertidumbre inerradicable, resolviendo en cada instante el problema de la escasez, todo en un tiempo empresarial, en la que si aciertan, obtendrán beneficios y si cometen errores, perdidas. (Ravier, 2016)

Al usar las verdades axiomáticas presentes en la teoría de la función empresarial, los hechos sociales analizados, aportan al conocimiento de la historia económica del Ecuador de los últimos siete años. No se considera esta investigación un aporte a la ciencia, pues los datos estadísticos lo que muestran es el resultado de la función empresarial y su aporte es a la historia económica de las empresas ecuatorianas, que preferiblemente las definimos como firmas.

Es muy difícil querer hacer un análisis de la formación de los precios a nivel de cada empresa, pues las variables implicadas deben ser muchas. En todo caso, por ejemplo en el sector petrolero se conoce que la tarifa para extracción de petróleo es establecida por un contrato de prestación de servicios entre la firma y el Estado, por lo mismo si el resultado de la capitalización de la empresa es alto, se podría decir que la firma aprovechó el poder del mercado para alcanzar altos niveles de capitalización y por tanto de formación de activos, esto como un caso de captura de rentas en la típica ineficiencia del Estado en materia de negocios.

\section{Resultados}

Un primer análisis fue calcular el peso, es decir la participación de esas cien firmas en el PIB sectorial, así como la participación del PIB sectorial en el PIB total. Para comparar el desempeño del sector económico del PIB con el de las firmas que se pertenecen a ese sector de acuerdo al clasificador internacional industrial uniforme CIIU. Para esto hemos usado la tasa de crecimiento del ingreso real como el diferencial que resulta de comparar un valor del año $n+1$, dividido para el año n, todo menos 1 y el resultado se multiplica por 100. Esto ha permitido calcular tanto el crecimiento económico porcentual, como el crecimiento de los ingresos reales de las firmas, estimación en la que se deflactaron sus ingresos. Se obtuvieron los siguientes resultados: 


\section{Tabla 1}

Desempeño de cien firmas según ranking oficial de la Superintendencia de Compañías por ventas o ingreso e impacto en el crecimiento de la economía ecuatoriana durante el periodo 2010-2016

\begin{tabular}{|c|c|c|c|c|c|}
\hline Sectores Económicos & $\begin{array}{l}\quad \text { Participación } \\
\text { en el PIB del } \\
\text { Sector } \\
\text { económico }\end{array}$ & $\begin{array}{l}\text { No de firmas } \\
\text { seleccionadas } \\
\text { según ranking }\end{array}$ & $\begin{array}{l}\text { Participación } \\
\text { de los ingresos } \\
\text { de las firmas con } \\
\text { relación al PIB } \\
\text { sectorial }\end{array}$ & $\begin{array}{l}\text { Crecimiento } \\
\text { del sector } \\
\text { económico }\end{array}$ & $\begin{array}{l}\text { Crecimiento } \\
\text { de los ingresos } \\
\text { de las firmas } \\
\text { seleccionadas }\end{array}$ \\
\hline $\begin{array}{l}\text { Manufactura (excepto } \\
\text { refinación de petróleo) }\end{array}$ & $11,8 \%$ & 35 & $78,1 \%$ & $2,3 \%$ & $-1,4 \%$ \\
\hline Comercio & $10,2 \%$ & 34 & $119,6 \%$ & $2,2 \%$ & $-0,8 \%$ \\
\hline Petróleo y minas & $9,9 \%$ & 5 & $24,2 \%$ & $2,1 \%$ & $40,0 \%$ \\
\hline Construcción & $9,4 \%$ & 7 & $9,8 \%$ & $5,1 \%$ & $17,7 \%$ \\
\hline $\begin{array}{l}\text { Enseñanza y Servicios } \\
\text { sociales y de salud }\end{array}$ & $8,4 \%$ & 1 & $1,7 \%$ & $3,3 \%$ & $4,0 \%$ \\
\hline $\begin{array}{l}\text { Agricultura, ganadería, } \\
\text { caza y silvicultura }\end{array}$ & $7,6 \%$ & 4 & $9,5 \%$ & $3,0 \%$ & $6,7 \%$ \\
\hline $\begin{array}{l}\text { Transporte } \\
\text { Correo y }\end{array}$ & $6,7 \%$ & 2 & $7,9 \%$ & $3,9 \%$ & $5,8 \%$ \\
\hline $\begin{array}{l}\text { Comunicaciones } \\
\text { Suministro de }\end{array}$ & $3,4 \%$ & 3 & $109,8 \%$ & $3,8 \%$ & $3,9 \%$ \\
\hline electricidad y agua & $2,3 \%$ & 1 & $11,9 \%$ & $11,2 \%$ & $-3,3 \%$ \\
\hline $\begin{array}{l}\text { Alojamiento y } \\
\text { servicios de comida } \\
\text { Acuicultura y pesca de }\end{array}$ & $1,7 \%$ & 1 & $9,5 \%$ & $2,0 \%$ & $2,4 \%$ \\
\hline $\begin{array}{l}\text { camarón } \\
\text { Pesca (excepto }\end{array}$ & $0,7 \%$ & 5 & $142,9 \%$ & $15,4 \%$ & $58,9 \%$ \\
\hline camarón) & $0,6 \%$ & 2 & $33,1 \%$ & $2,8 \%$ & $1,7 \%$ \\
\hline $\begin{array}{l}\text { Sectores considerados } \\
\text { del PIB y relación con } \\
\text { las firmas seleccionadas }\end{array}$ & $73 \%$ & 100 & $44 \%$ & $3,3 \%$ & $12,3 \%$ \\
\hline PIB TOTAL & $100 \%$ & & $32 \%$ & $3,0 \%$ & \\
\hline
\end{tabular}

Nota: Recuperado del boletín estadística mensual No. 1988 de octubre 2017del Banco Central del Ecuador, cuadro

IEM-432-e PIB por industria: https://www.bce.fin.ec/index.php/component/k2/item/776 y de la información constante en el ranking de Superintendencia de Compañías por los años 2010 a 2017

https://appscvs.supercias.gob.ec/rankingCias/ Los datos han sido trabajados por los autores con base a la información indicada.

Un segundo análisis presenta el impacto en el pleno empleo de las 100 firmas consideradas, teniendo en cuenta que este empleo es del tipo no precario, por tanto, está sujeto al cumplimiento de toda la normativa constante en las leyes y reglamentos sobre las relaciones laborales, por ejemplo, la aportación al sistema de previsión social y al sistema de rentas internas con todo lo concerniente al régimen tributario interno, lo que podría llamarse empleo formal. El impacto resultante es relevante según se aprecia a continuación: 


\section{Tabla 2}

Desempeño de cien firmas según ranking oficial de la Superintendencia de Compañías por creación de empleos y su relación con el empleo total entre 2010 y 2016

\begin{tabular}{|c|c|c|c|c|}
\hline Sectores Económicos & $\begin{array}{l}\text { No de firmas } \\
\text { seleccionadas según } \\
\text { ranking }\end{array}$ & $\begin{array}{l}\text { Empleados } \\
\text { por estas } \\
\text { firmas en } 2010\end{array}$ & $\begin{array}{l}\text { Empleados } \\
\text { por estas } \\
\text { firmas en } 2016\end{array}$ & $\begin{array}{l}\text { Nuevos } \\
\text { empleos o } \\
\text { reducciones de } \\
\text { empleos de estas } \\
\text { firmas }\end{array}$ \\
\hline \multicolumn{5}{|l|}{ Manufactura (excepto refinación de } \\
\hline Comercio & 34 & 39.689 & 51.049 & 11.360 \\
\hline Petróleo y minas & 5 & 1.272 & 2.548 & 1.276 \\
\hline Construcción & 7 & 3.530 & 10.831 & 7.301 \\
\hline $\begin{array}{l}\text { Enseñanza y Servicios sociales y } \\
\text { de salud } \\
\text { Agricultura, ganadería, caza y }\end{array}$ & 1 & 818 & 830 & 12 \\
\hline silvicultura & 4 & 14.211 & 8.804 & -5.407 \\
\hline Transporte & 2 & 2.089 & 2.128 & 39 \\
\hline Correo y Comunicaciones & 3 & 3.615 & 4.795 & 1.180 \\
\hline Suministro de electricidad y agua & 1 & 1.211 & 1.154 & -57 \\
\hline Alojamiento y servicios de comida & 1 & 2.607 & 4.165 & 1.558 \\
\hline Acuicultura y pesca de camarón & 5 & 1.534 & 7.566 & 6.032 \\
\hline Pesca (excepto camarón) & 2 & 2.053 & 1.678 & -375 \\
\hline \multicolumn{5}{|l|}{ Empleo pleno de las 100 firmas por } \\
\hline \multicolumn{2}{|c|}{ Empleo pleno total del Ecuador, No de personas } & 2.875 .533 & 3.243 .293 & 367.760 \\
\hline \multicolumn{2}{|c|}{ Impacto en el pleno empleo por parte de las cien firmas } & $4,0 \%$ & $4,9 \%$ & $11,5 \%$ \\
\hline
\end{tabular}

Nota: Recuperado de la información constante en el ranking de Superintendencia de Compañías por los años 2010 a

2017 https://appscvs.supercias.gob.ec/rankingCias/ y de la información del Instituto Nacional de Estadística y

Censos http://www.ecuadorencifras.gob.ec/documentos/web-inec/EMPLEO/2018/Junio-

2018/062018_Mercado\%20Laboral.pdf (INEC, 2018).- La comparación ha sido elaborada por los autores considerando la información de inicio y fin de período.

El tercer análisis prepara la información estadística de estas firmas sobre el crecimiento de punta a punta de los activos de las empresas y comprueba el impacto por comparación en la formación bruta de capital fijo FBCF tanto del total del sector privado, como del sector al que se corresponden estas firmas. Nuevamente se aprecia un peso significativo del aporte de estas cien firmas a los niveles de inversión o FBCF 


\section{Tabla 3}

Desempeño de cien firmas según ranking oficial de la Superintendencia de Compañías por crecimiento de la inversión de acuerdo al incremento de los activos entre 2010 y $2016^{*}$

\begin{tabular}{llllll} 
& No de & Activos a 2010 & \multicolumn{1}{c}{ Activos a } & Incremento de & Tasa de \\
Sectores Económicos a valores & los Activos en & variación \\
& firmas & a valores & constantes en & los \\
seleccionadas & constantes en & millones de & promedio \\
& según ranking & millones de USD & USD & USD & anual \\
& & &
\end{tabular}

\begin{tabular}{|c|c|c|c|c|c|}
\hline $\begin{array}{l}\text { Manufactura (excepto } \\
\text { refinación de petróleo) }\end{array}$ & 35 & 3.908 & 4.778 & 870 & $2,9 \%$ \\
\hline Comercio & 34 & 2.621 & 4.378 & 1.758 & $7,6 \%$ \\
\hline Petróleo y minas & 5 & 1.670 & 9.987 & 8.318 & $29,1 \%$ \\
\hline Construcción & 7 & 262 & 1.238 & 976 & $24,8 \%$ \\
\hline $\begin{array}{l}\text { Enseñanza y Servicios } \\
\text { sociales y de salud }\end{array}$ & 1 & 16 & 23 & 8 & $5,8 \%$ \\
\hline $\begin{array}{l}\text { Agricultura, ganadería, caza } \\
\text { y silvicultura }\end{array}$ & 4 & 376 & 480 & 103 & $3,5 \%$ \\
\hline Transporte & 2 & 256 & 374 & 118 & $5,6 \%$ \\
\hline Correo y Comunicaciones & 3 & 1.882 & 2.957 & 1.075 & $6,7 \%$ \\
\hline $\begin{array}{l}\text { Suministro de electricidad y } \\
\text { agua }\end{array}$ & 1 & 247 & 362 & 115 & $5,6 \%$ \\
\hline $\begin{array}{l}\text { Alojamiento y servicios de } \\
\text { comida }\end{array}$ & 1 & 45 & 48 & 3 & $1,1 \%$ \\
\hline $\begin{array}{l}\text { Acuicultura y pesca de } \\
\text { camarón }\end{array}$ & 5 & 99 & 885 & 786 & $36,8 \%$ \\
\hline Pesca (excepto camarón) & 2 & 82 & 76 & -6 & $-1,0 \%$ \\
\hline $\begin{array}{l}\text { Número de firmas } \\
\text { consideradas y monto de sus } \\
\text { activos a inicio y fin de } \\
\text { período }\end{array}$ & 100 & 11.463 & 25.587 & 14.124 & $12,2 \%$ \\
\hline $\begin{array}{l}\text { Formación Bruta de capital } \\
\text { de toda la economía en el sec } \\
\text { Impacto en la inversión por }\end{array}$ & $\begin{array}{l}\text { por to } \\
\text { rivad } \\
\text { e de }\end{array}$ & do a niv & & 67.476 & \\
\hline firmas & & & & $20,9 \%$ & \\
\hline
\end{tabular}

El cuarto análisis es apenas una aproximación del comportamiento de la evolución de los precios de los bienes y servicios finales producidos por los diferentes sectores económicos bajo estudio, comparados con el crecimiento promedio anual de la inflación de toda la economía en su conjunto, medida con base a la evolución del deflactor del PIB. El deflactor no es sino un índice 
de precios de base 100, que se construye dividiendo el PIB a valores de cada año para el PIB a valores constantes.

\section{Tabla 4}

Inflación resultante del deflactor sectorial calculado e impacto en el crecimiento de los precios de la economía ecuatoriana durante el periodo 2010-2016

\begin{tabular}{|c|c|c|c|c|c|}
\hline Sectores Económicos & $\begin{array}{l}\quad \text { deflactor } \\
\text { calculado } \\
\text { para el año } \\
2010\end{array}$ & $\begin{array}{l}\quad \text { deflactor } \\
\text { calculado } \\
\text { para el año } \\
2016\end{array}$ & $\begin{array}{l}\text { crecimiento } \\
\text { acumulado de } \\
\text { los precios } \\
\text { sectoriales }\end{array}$ & $\begin{array}{l}\text { crecimiento } \\
\text { promedio } \\
\text { anual }\end{array}$ & \begin{tabular}{l}
\multicolumn{1}{c}{ puntos } \\
porcentuales \\
sobre la inflación \\
promedio con \\
base al deflactor
\end{tabular} \\
\hline
\end{tabular}

\begin{tabular}{|c|c|c|c|c|c|}
\hline $\begin{array}{l}\text { Manufactura (excepto } \\
\text { refinación de petróleo) }\end{array}$ & 125,20 & 169,80 & 44,60 & $4,4 \%$ & $2,3 \%$ \\
\hline Comercio & 122,80 & 140,60 & 17,80 & $2,0 \%$ & $-0,2 \%$ \\
\hline Petróleo y minas & 127,20 & 55,60 & $-71,60$ & $-11,2 \%$ & $-13,3 \%$ \\
\hline Construcción & 139,80 & 183,10 & 43,30 & $3,9 \%$ & $1,8 \%$ \\
\hline $\begin{array}{l}\text { Enseñanza y Servicios } \\
\text { sociales y de salud }\end{array}$ & 119,80 & 144,70 & 24,90 & $2,7 \%$ & $0,6 \%$ \\
\hline $\begin{array}{l}\text { Agricultura, ganadería, } \\
\text { caza y silvicultura }\end{array}$ & 139,20 & 156,60 & 17,40 & $1,7 \%$ & $-0,4 \%$ \\
\hline Transporte & 99,80 & 106,70 & 6,90 & $1,0 \%$ & $-1,2 \%$ \\
\hline Correo y Comunicaciones & 91,90 & 83,80 & $-8,10$ & $-1,3 \%$ & $-3,5 \%$ \\
\hline $\begin{array}{l}\text { Suministro de electricidad } \\
\text { y agua }\end{array}$ & 81,80 & 83,60 & 1,80 & $0,3 \%$ & $-1,8 \%$ \\
\hline $\begin{array}{l}\text { Alojamiento y servicios } \\
\text { de comida }\end{array}$ & 127,30 & 182,60 & 55,30 & $5,3 \%$ & $3,1 \%$ \\
\hline $\begin{array}{l}\text { Acuicultura y pesca de } \\
\text { camarón }\end{array}$ & 115,70 & 73,60 & $-42,10$ & $-6,3 \%$ & $-8,4 \%$ \\
\hline Pesca (excepto camarón) & 113,60 & 134,10 & 20,50 & $2,4 \%$ & $0,3 \%$ \\
\hline $\begin{array}{l}\text { Sectores considerados del } \\
\text { PIB y relación con las } \\
\text { firmas seleccionadas }\end{array}$ & 123,10 & 142,80 & 19,70 & $2,1 \%$ & \\
\hline
\end{tabular}

El último análisis es de tipo cualitativo e implicó el haber obtenido información de las propias empresas, muchas de las cuales presentan esta información en directorios empresariales y revistas especializadas. Como se aprecia a continuación en la Tabla 5 en su gran mayoría estas firmas dan a conocer su estrategia competitiva basándose en el estándar estratégico que les permite diferenciarse y liderar el mercado en su respectiva industria, presentando en muchos de los casos el diferenciador de marca, que permite a sus clientes y consumidores el reconocimiento y fidelización. 
Tabla 5

Las 100 firmas y su registro estratégico por sectores

\begin{tabular}{|c|c|c|c|c|c|c|c|c|c|}
\hline No & Nombre & $\begin{array}{c}\text { Sector } \\
\text { Económico }\end{array}$ & Provincia & Historia & Misión & Visión & $\begin{array}{c}\text { Estrategia } \\
\text { competitiva }\end{array}$ & $\begin{array}{l}\text { Diferenciación } \\
\text { en el mercado }\end{array}$ & $\begin{array}{c}\text { Atributos de } \\
\text { la Marca }\end{array}$ \\
\hline 1 & $\begin{array}{l}\text { Unión de } \\
\text { Bananeros } \\
\text { Ecuatorianos } \\
\text { S.A. Ubesa }\end{array}$ & Agricultura & Guayas & $\mathrm{x}$ & $\mathrm{x}$ & $\mathrm{x}$ & $\mathrm{x}$ & $\mathrm{x}$ & $\mathrm{x}$ \\
\hline 2 & $\begin{array}{l}\text { Reybanpac Rey } \\
\text { Banano del } \\
\text { Pacífico C.A }\end{array}$ & Agricultura & Guayas & $\mathrm{x}$ & $\mathrm{x}$ & $\mathrm{x}$ & $\mathrm{x}$ & $\mathrm{x}$ & $\mathrm{x}$ \\
\hline 3 & Truisfruit S.A. & Agricultura & Guayas & $\mathrm{x}$ & $\mathrm{x}$ & $\mathrm{x}$ & $\mathrm{x}$ & $\mathrm{x}$ & $\mathrm{x}$ \\
\hline 4 & $\begin{array}{l}\text { Sabrostar Fruit } \\
\text { Company S.A. }\end{array}$ & Agricultura & Guayas & $\mathrm{x}$ & $\mathrm{X}$ & $\mathrm{x}$ & $\mathrm{x}$ & $\mathrm{x}$ & $\mathrm{x}$ \\
\hline 5 & $\begin{array}{l}\text { Salica del } \\
\text { Ecuador s.a. }\end{array}$ & Pesca & Guayas & $\mathrm{x}$ & $\mathrm{x}$ & $\mathrm{x}$ & $\mathrm{x}$ & $\mathrm{x}$ & $\mathrm{x}$ \\
\hline 6 & $\begin{array}{l}\text { Operadora y } \\
\text { Procesadora de } \\
\text { Productos } \\
\text { Marinos Omarsa } \\
\text { S.A. }\end{array}$ & $\begin{array}{l}\text { Acuicultura } \\
\text { y pesca de } \\
\text { camarón }\end{array}$ & Guayas & $\mathrm{x}$ & $\mathrm{x}$ & $\mathrm{x}$ & $\mathrm{x}$ & $\mathrm{x}$ & $\mathrm{x}$ \\
\hline 7 & $\begin{array}{l}\text { Sociedad } \\
\text { Nacional de } \\
\text { Galápagos C.A }\end{array}$ & $\begin{array}{l}\text { Acuicultura } \\
\text { y pesca de } \\
\text { camarón }\end{array}$ & Guayas & $\mathrm{x}$ & $\mathrm{x}$ & $\mathrm{x}$ & $\mathrm{x}$ & $\mathrm{x}$ & $\mathrm{x}$ \\
\hline 8 & Promarisco S.A & $\begin{array}{l}\text { Acuicultura } \\
\text { y pesca de } \\
\text { camarón }\end{array}$ & Guayas & $\mathrm{x}$ & $\mathrm{x}$ & $\mathrm{x}$ & $\mathrm{x}$ & $\mathrm{x}$ & $\mathrm{x}$ \\
\hline 9 & $\begin{array}{l}\text { Vitapro Ecuador } \\
\text { Cía. Ltda. }\end{array}$ & $\begin{array}{l}\text { Acuicultura } \\
\text { y pesca de } \\
\text { camarón }\end{array}$ & Guayas & $\mathrm{x}$ & $\mathrm{x}$ & $\mathrm{x}$ & $\mathrm{x}$ & $\mathrm{x}$ & $\mathrm{x}$ \\
\hline 10 & $\begin{array}{l}\text { Empacadora } \\
\text { Grupo Granmar } \\
\text { S.A. Empagran }\end{array}$ & $\begin{array}{l}\text { Acuicultura } \\
\text { y pesca de } \\
\text { camarón }\end{array}$ & Guayas & $\mathrm{x}$ & $\mathrm{x}$ & $\mathrm{x}$ & $\mathrm{x}$ & $\mathrm{x}$ & $\mathrm{x}$ \\
\hline 11 & $\begin{array}{l}\text { Consorcio } \\
\text { Shushufindi S.A. }\end{array}$ & $\begin{array}{l}\text { Petróleo y } \\
\text { minas }\end{array}$ & Pichincha & $\mathrm{x}$ & $\mathrm{X}$ & $\mathrm{x}$ & $\mathrm{x}$ & $\mathrm{x}$ & $\mathrm{x}$ \\
\hline 12 & $\begin{array}{l}\text { Shaya Ecuador } \\
\text { S.A }\end{array}$ & $\begin{array}{l}\text { Petróleo y } \\
\text { minas }\end{array}$ & Pichincha & $\mathrm{x}$ & $\mathrm{X}$ & $\mathrm{x}$ & $\mathrm{x}$ & $\mathrm{x}$ & $\mathrm{x}$ \\
\hline 13 & $\begin{array}{l}\text { Andes Petroleum } \\
\text { Ecuador Cía. } \\
\text { Ltda. }\end{array}$ & $\begin{array}{l}\text { Petróleo y } \\
\text { minas }\end{array}$ & Pichincha & $\mathrm{x}$ & $\mathrm{X}$ & $\mathrm{x}$ & $\mathrm{x}$ & $\mathrm{X}$ & $\mathrm{x}$ \\
\hline 14 & $\begin{array}{l}\text { Schlumberger } \\
\text { del Ecuador S.A. }\end{array}$ & $\begin{array}{l}\text { Petróleo y } \\
\text { minas }\end{array}$ & Pichincha & $\mathrm{x}$ & $\mathrm{X}$ & $\mathrm{x}$ & $\mathrm{x}$ & $\mathrm{x}$ & $\mathrm{x}$ \\
\hline 15 & $\begin{array}{l}\text { Oleoducto de } \\
\text { Crudos Pesados } \\
\text { (OCP) Ecuador } \\
\text { S. A. }\end{array}$ & $\begin{array}{l}\text { Petróleo y } \\
\text { minas }\end{array}$ & Pichincha & $\mathrm{x}$ & $\mathrm{X}$ & $\mathrm{x}$ & $\mathrm{x}$ & $\mathrm{x}$ & $\mathrm{x}$ \\
\hline
\end{tabular}




\begin{tabular}{|c|c|c|c|c|c|c|c|c|c|}
\hline No & Nombre & $\begin{array}{c}\text { Sector } \\
\text { Económico }\end{array}$ & Provincia & Historia & Misión & Visión & $\begin{array}{c}\text { Estrategia } \\
\text { competitiva }\end{array}$ & $\begin{array}{l}\text { Diferenciación } \\
\text { en el mercado }\end{array}$ & $\begin{array}{c}\text { Atributos de } \\
\text { la Marca }\end{array}$ \\
\hline 16 & $\begin{array}{l}\text { Agip Oil } \\
\text { Ecuador B.V. }\end{array}$ & $\begin{array}{l}\text { Petróleo y } \\
\text { minas }\end{array}$ & Pichincha & $\mathrm{x}$ & $\mathrm{x}$ & $\mathrm{x}$ & $\mathrm{x}$ & $\mathrm{x}$ & $\mathrm{x}$ \\
\hline 17 & $\begin{array}{l}\text { Procesadora } \\
\text { Nacional de } \\
\text { Alimentos C. A. } \\
\text { Pronaca }\end{array}$ & Manufactura & Pichincha & $\mathrm{x}$ & $\mathrm{x}$ & $\mathrm{x}$ & $\mathrm{x}$ & $\mathrm{x}$ & $\mathrm{x}$ \\
\hline 18 & $\begin{array}{l}\text { General Motors } \\
\text { del Ecuador S.A. }\end{array}$ & Manufactura & Pichincha & $\mathrm{x}$ & $\mathrm{x}$ & $\mathrm{x}$ & $\mathrm{x}$ & $\mathrm{x}$ & $\mathrm{x}$ \\
\hline 19 & $\begin{array}{l}\text { Arca Continental } \\
\text { S.A. Bursátil de } \\
\text { Capital Variable }\end{array}$ & Manufactura & Pichincha & $\mathrm{x}$ & $\mathrm{x}$ & $\mathrm{x}$ & $\mathrm{x}$ & $\mathrm{x}$ & $\mathrm{x}$ \\
\hline 20 & $\begin{array}{l}\text { Nestle Ecuador } \\
\text { s.a. }\end{array}$ & Manufactura & Pichincha & $\mathrm{x}$ & $\mathrm{x}$ & $\mathrm{x}$ & $\mathrm{x}$ & $\mathrm{x}$ & $\mathrm{x}$ \\
\hline 21 & $\begin{array}{l}\text { Cervecería } \\
\text { Nacional CN } \\
\text { S.A. }\end{array}$ & Manufactura & Guayas & $\mathrm{x}$ & $\mathrm{x}$ & $\mathrm{x}$ & $\mathrm{x}$ & $\mathrm{x}$ & $\mathrm{x}$ \\
\hline 22 & La Fabril S.A. & Manufactura & Manabí & $\mathrm{x}$ & $\mathrm{x}$ & $\mathrm{x}$ & $\mathrm{x}$ & $\mathrm{x}$ & $\mathrm{x}$ \\
\hline 23 & $\begin{array}{l}\text { Holcim Ecuador } \\
\text { S.A. }\end{array}$ & Manufactura & Guayas & $\mathrm{x}$ & $\mathrm{x}$ & $\mathrm{x}$ & $\mathrm{x}$ & $\mathrm{x}$ & $\mathrm{x}$ \\
\hline 24 & $\begin{array}{l}\text { Industrial } \\
\text { Pesquera Santa } \\
\text { Priscila S.A. }\end{array}$ & Manufactura & Guayas & $\mathrm{x}$ & $\mathrm{x}$ & $\mathrm{x}$ & $\mathrm{x}$ & $\mathrm{x}$ & $\mathrm{x}$ \\
\hline 25 & $\begin{array}{l}\text { Omnibus BB } \\
\text { Transportes S.A. }\end{array}$ & Manufactura & Pichincha & $\mathrm{x}$ & $\mathrm{x}$ & $\mathrm{x}$ & $\mathrm{x}$ & $\mathrm{x}$ & $\mathrm{x}$ \\
\hline 26 & $\begin{array}{l}\text { Negocios } \\
\text { Industriales Real } \\
\text { N.I.R.S.A. S.A. }\end{array}$ & Manufactura & Guayas & $\mathrm{x}$ & $\mathrm{x}$ & $\mathrm{x}$ & $\mathrm{x}$ & $\mathrm{x}$ & $\mathrm{x}$ \\
\hline 27 & $\begin{array}{l}\text { Unilever Andina } \\
\text { Ecuador S.A. }\end{array}$ & Manufactura & Guayas & $\mathrm{x}$ & $\mathrm{x}$ & $\mathrm{x}$ & $\mathrm{x}$ & $\mathrm{x}$ & $\mathrm{x}$ \\
\hline 28 & $\begin{array}{l}\text { Bebidas Arca } \\
\text { Continental } \\
\text { Ecuador Arcador } \\
\text { S.A. }\end{array}$ & Manufactura & Pichincha & $\mathrm{x}$ & $\mathrm{x}$ & $\mathrm{x}$ & $\mathrm{x}$ & $\mathrm{x}$ & $\mathrm{x}$ \\
\hline 29 & $\begin{array}{l}\text { Acería del } \\
\text { Ecuador C. A. } \\
\text { ADELCA }\end{array}$ & Manufactura & Pichincha & $\mathrm{x}$ & $\mathrm{x}$ & $\mathrm{x}$ & $\mathrm{x}$ & $\mathrm{x}$ & $\mathrm{x}$ \\
\hline 30 & $\begin{array}{l}\text { Industrial Danec } \\
\text { S.A. }\end{array}$ & Manufactura & Pichincha & $\mathrm{x}$ & $\mathrm{x}$ & $\mathrm{x}$ & $\mathrm{x}$ & $\mathrm{x}$ & $\mathrm{x}$ \\
\hline 31 & $\begin{array}{l}\text { The Tesalia } \\
\text { Springs } \\
\text { Company S.A. }\end{array}$ & Manufactura & Pichincha & $\mathrm{x}$ & $\mathrm{x}$ & $\mathrm{x}$ & & $\mathrm{x}$ & $\mathrm{x}$ \\
\hline 32 & Novacero S.A. & Manufactura & Cotopaxi & $\mathrm{x}$ & $\mathrm{x}$ & $\mathrm{x}$ & & & $\mathrm{x}$ \\
\hline 33 & $\begin{array}{l}\text { Ecuaquimica } \\
\text { Ecuatoriana de } \\
\text { Productos } \\
\text { Químicos C.A. }\end{array}$ & Manufactura & Guayas & $\mathrm{x}$ & $\mathrm{x}$ & $\mathrm{x}$ & $\mathrm{x}$ & $\mathrm{x}$ & \\
\hline
\end{tabular}




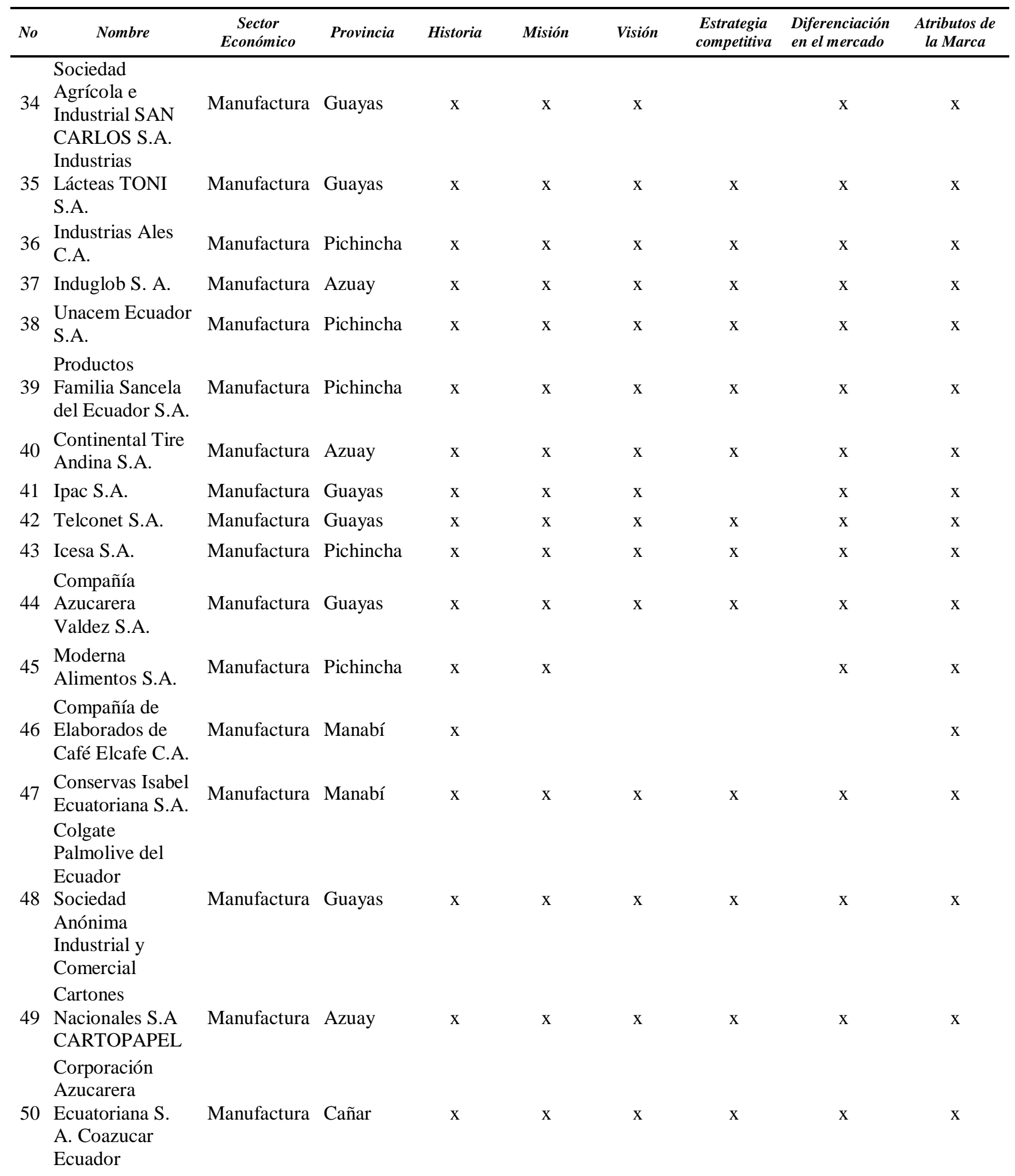




\begin{tabular}{|c|c|c|c|c|c|c|c|c|c|}
\hline No & Nombre & $\begin{array}{c}\text { Sector } \\
\text { Económico }\end{array}$ & Provincia & Historia & Misión & Visión & $\begin{array}{l}\text { Estrategia } \\
\text { competitiva }\end{array}$ & $\begin{array}{l}\text { Diferenciación } \\
\text { en el mercado }\end{array}$ & $\begin{array}{l}\text { Atributos de } \\
\text { la Marca }\end{array}$ \\
\hline 51 & $\begin{array}{l}\text { Kimberly - Clark } \\
\text { Ecuador S.A. }\end{array}$ & Manufactura & Guayas & $\mathrm{x}$ & $\mathrm{x}$ & $\mathrm{x}$ & $\mathrm{x}$ & $\mathrm{x}$ & $\mathrm{x}$ \\
\hline 52 & $\begin{array}{l}\text { International } \\
\text { Water Services } \\
\text { (Guayaquil) } \\
\text { Interagua Cía. } \\
\text { Ltda. }\end{array}$ & $\begin{array}{l}\text { Suministro } \\
\text { de } \\
\text { electricidad } \\
\text { y agua }\end{array}$ & Guayas & $\mathrm{x}$ & $\mathrm{x}$ & $\mathrm{x}$ & $\mathrm{x}$ & $\mathrm{x}$ & $\mathrm{x}$ \\
\hline 53 & $\begin{array}{l}\text { China Camc } \\
\text { Engineering Co. } \\
\text { Ltda. }\end{array}$ & Construcción & Pichincha & $\mathrm{x}$ & $\mathrm{x}$ & $\mathrm{x}$ & $\mathrm{x}$ & $\mathrm{x}$ & $\mathrm{x}$ \\
\hline 54 & $\begin{array}{l}\text { Hidalgo e } \\
\text { Hidalgo S. A. } \\
\text { Panamericana }\end{array}$ & Construcción & Pichincha & $\mathrm{x}$ & $\mathrm{x}$ & $\mathrm{x}$ & $\mathrm{x}$ & $\mathrm{x}$ & $\mathrm{x}$ \\
\hline 55 & $\begin{array}{l}\text { Vial S.A. } \\
\text { Panavial }\end{array}$ & Construcción & Pichincha & $\mathrm{x}$ & $\mathrm{x}$ & $\mathrm{x}$ & $\mathrm{x}$ & $\mathrm{x}$ & $\mathrm{x}$ \\
\hline 56 & $\begin{array}{l}\text { Sinohydro } \\
\text { Corporation } \\
\text { Limited }\end{array}$ & Construcción & Pichincha & $\mathrm{x}$ & $\mathrm{x}$ & $\mathrm{x}$ & $\mathrm{x}$ & $\mathrm{x}$ & $\mathrm{x}$ \\
\hline 57 & $\begin{array}{l}\text { "Consorcio } \\
\text { Línea 1" - Metro } \\
\text { de Quito: } \\
\text { Acciona }\end{array}$ & Construcción & Pichincha & $\mathrm{x}$ & $\mathrm{x}$ & $\mathrm{x}$ & $\mathrm{x}$ & $\mathrm{x}$ & $\mathrm{x}$ \\
\hline 58 & $\begin{array}{l}\text { Sinohydro } \\
\text { Corporation }\end{array}$ & Construcción & Pichincha & & & & & & \\
\hline 59 & $\begin{array}{l}\text { Harbin Electric } \\
\text { International } \\
\text { Co., Ltda. }\end{array}$ & Construcción & Pichincha & $\mathrm{x}$ & $\mathrm{x}$ & $\mathrm{x}$ & $\mathrm{x}$ & $\mathrm{x}$ & $\mathrm{x}$ \\
\hline 60 & $\begin{array}{l}\text { Corporación } \\
\text { Favorita C.A. }\end{array}$ & Comercio & Pichincha & $\mathrm{x}$ & $\mathrm{x}$ & $\mathrm{x}$ & $\mathrm{x}$ & $\mathrm{x}$ & $\mathrm{x}$ \\
\hline 61 & $\begin{array}{l}\text { Corporación El } \\
\text { Rosado S.A. }\end{array}$ & Comercio & Guayas & $\mathrm{x}$ & $\mathrm{x}$ & $\mathrm{x}$ & $\mathrm{x}$ & $\mathrm{x}$ & $\mathrm{x}$ \\
\hline 62 & Dinadec S.A. & Comercio & Guayas & $\mathrm{x}$ & $\mathrm{x}$ & $\mathrm{x}$ & $\mathrm{x}$ & $\mathrm{x}$ & $\mathrm{x}$ \\
\hline 63 & $\begin{array}{l}\text { Distribuidora } \\
\text { Farmacéutica } \\
\text { Ecuatoriana } \\
\text { (Difare) S.A. }\end{array}$ & Comercio & Guayas & $\mathrm{x}$ & $\mathrm{x}$ & $\mathrm{x}$ & $\mathrm{x}$ & $\mathrm{x}$ & $\mathrm{x}$ \\
\hline 64 & $\begin{array}{l}\text { Tiendas } \\
\text { Industriales } \\
\text { Asociadas Tía } \\
\text { S.A. }\end{array}$ & Comercio & Guayas & $\mathrm{x}$ & $\mathrm{x}$ & $\mathrm{x}$ & $\mathrm{x}$ & $\mathrm{x}$ & $\mathrm{x}$ \\
\hline 65 & $\begin{array}{l}\text { Primax } \\
\text { Comercial del } \\
\text { Ecuador S.A. }\end{array}$ & Comercio & Pichincha & $\mathrm{x}$ & $\mathrm{x}$ & $\mathrm{x}$ & $\mathrm{x}$ & $\mathrm{x}$ & $\mathrm{x}$ \\
\hline 66 & $\begin{array}{l}\text { Petróleos y } \\
\text { Servicios PYS } \\
\text { C.A. }\end{array}$ & Comercio & Pichincha & $\mathrm{x}$ & $\mathrm{x}$ & $\mathrm{x}$ & $\mathrm{x}$ & $\mathrm{x}$ & $\mathrm{x}$ \\
\hline 67 & $\begin{array}{l}\text { Construmercado } \\
\text { S.A. }\end{array}$ & Comercio & Guayas & $\mathrm{x}$ & $\mathrm{x}$ & $\mathrm{x}$ & $\mathrm{x}$ & $\mathrm{x}$ & $\mathrm{x}$ \\
\hline
\end{tabular}




\begin{tabular}{|c|c|c|c|c|c|c|c|c|c|}
\hline No & Nombre & $\begin{array}{c}\text { Sector } \\
\text { Económico }\end{array}$ & Provincia & Historia & Misión & Visión & $\begin{array}{c}\text { Estrategia } \\
\text { competitiva }\end{array}$ & $\begin{array}{l}\text { Diferenciación } \\
\text { en el mercado }\end{array}$ & $\begin{array}{c}\text { Atributos de } \\
\text { la Marca }\end{array}$ \\
\hline 68 & $\begin{array}{l}\text { Distribuidora } \\
\text { Importadora } \\
\text { Dipor S.A. }\end{array}$ & Comercio & Guayas & $\mathrm{x}$ & $\mathrm{x}$ & $\mathrm{x}$ & $\mathrm{x}$ & $\mathrm{x}$ & $\mathrm{x}$ \\
\hline 69 & $\begin{array}{l}\text { Leterago del } \\
\text { Ecuador S.A. }\end{array}$ & Comercio & Pichincha & $\mathrm{x}$ & $\mathrm{x}$ & $\mathrm{x}$ & $\mathrm{x}$ & $\mathrm{x}$ & $\mathrm{x}$ \\
\hline 70 & $\begin{array}{l}\text { Mega Santamaría } \\
\text { S.A. }\end{array}$ & Comercio & Pichincha & $\mathrm{x}$ & $\mathrm{x}$ & $\mathrm{x}$ & $\mathrm{x}$ & $\mathrm{x}$ & $\mathrm{x}$ \\
\hline 71 & $\begin{array}{l}\text { Proveedora } \\
\text { Ecuatoriana S.A. } \\
\text { PROESA }\end{array}$ & Comercio & Pichincha & $\mathrm{x}$ & $\mathrm{x}$ & $\mathrm{x}$ & $\mathrm{x}$ & $\mathrm{x}$ & $\mathrm{x}$ \\
\hline 72 & $\begin{array}{l}\text { Farmaenlace Cía. } \\
\text { Ltda. }\end{array}$ & Comercio & Pichincha & $\mathrm{x}$ & $\mathrm{x}$ & $\mathrm{x}$ & $\mathrm{x}$ & $\mathrm{x}$ & $\mathrm{x}$ \\
\hline 73 & Agripac S.A. & Comercio & Guayas & $\mathrm{x}$ & $\mathrm{x}$ & $\mathrm{x}$ & $\mathrm{x}$ & $\mathrm{x}$ & $\mathrm{x}$ \\
\hline 74 & Econofarm S.A. & Comercio & Pichincha & $\mathrm{x}$ & $\mathrm{x}$ & $\mathrm{x}$ & $\mathrm{x}$ & $\mathrm{x}$ & $\mathrm{x}$ \\
\hline 75 & $\begin{array}{l}\text { Gerardo Ortiz e } \\
\text { Hijos Cía. Ltda. }\end{array}$ & Comercio & Guayas & $\mathrm{x}$ & $\mathrm{x}$ & $\mathrm{x}$ & $\mathrm{x}$ & $\mathrm{x}$ & $\mathrm{x}$ \\
\hline 76 & $\begin{array}{l}\text { Almacenes de } \\
\text { Prati S.A. }\end{array}$ & Comercio & Guayas & $\mathrm{x}$ & $\mathrm{x}$ & $\mathrm{x}$ & $\mathrm{x}$ & $\mathrm{x}$ & $\mathrm{x}$ \\
\hline 77 & Atimasa S.A. & Comercio & Guayas & $\mathrm{x}$ & $\mathrm{x}$ & $\mathrm{x}$ & $\mathrm{x}$ & $\mathrm{x}$ & $\mathrm{x}$ \\
\hline 78 & $\begin{array}{l}\text { Comercial Kywi } \\
\text { S.A. }\end{array}$ & Comercio & Pichincha & $\mathrm{x}$ & $\mathrm{x}$ & $\mathrm{x}$ & $\mathrm{x}$ & $\mathrm{x}$ & $\mathrm{x}$ \\
\hline 79 & Gisis S.A. & Comercio & Guayas & $\mathrm{x}$ & $\mathrm{x}$ & $\mathrm{x}$ & $\mathrm{x}$ & $\mathrm{x}$ & $\mathrm{x}$ \\
\hline 80 & Quifatex S.A. & Comercio & Pichincha & $\mathrm{x}$ & $\mathrm{x}$ & $\mathrm{x}$ & $\mathrm{x}$ & $\mathrm{x}$ & $\mathrm{x}$ \\
\hline 81 & Aekia S.A. & Comercio & Pichincha & $\mathrm{x}$ & $\mathrm{x}$ & $\mathrm{x}$ & $\mathrm{x}$ & $\mathrm{x}$ & $\mathrm{x}$ \\
\hline 82 & $\begin{array}{l}\text { Petróleos de los } \\
\text { Ríos Petrolríos } \\
\text { C.A. }\end{array}$ & Comercio & $\begin{array}{l}\text { Santo } \\
\text { domingo } \\
\text { de los } \\
\text { Tsáchilas }\end{array}$ & $\mathrm{x}$ & $\mathrm{x}$ & $\mathrm{x}$ & $\mathrm{x}$ & $\mathrm{x}$ & $\mathrm{x}$ \\
\hline 83 & $\begin{array}{l}\text { Exxonmobil } \\
\text { Ecuador Cía. } \\
\text { Ltda. }\end{array}$ & Comercio & Pichincha & $\mathrm{x}$ & & $\mathrm{x}$ & $\mathrm{x}$ & $\mathrm{x}$ & $\mathrm{x}$ \\
\hline 84 & Marcimex S.A. & Comercio & Azuay & $\mathrm{x}$ & $\mathrm{x}$ & $\mathrm{x}$ & $\mathrm{x}$ & $\mathrm{x}$ & $\mathrm{x}$ \\
\hline 85 & $\begin{array}{l}\text { Yanbal Ecuador } \\
\text { S.A. }\end{array}$ & Comercio & Pichincha & $\mathrm{x}$ & $\mathrm{x}$ & $\mathrm{x}$ & $\mathrm{x}$ & $\mathrm{x}$ & $\mathrm{x}$ \\
\hline 86 & $\begin{array}{l}\text { Lutexsa } \\
\text { Industrial } \\
\text { Comercial Cía. } \\
\text { Ltda. }\end{array}$ & Comercio & Guayas & $\mathrm{x}$ & & & & & \\
\hline 87 & $\begin{array}{l}\text { Compañía } \\
\text { General de } \\
\text { Comercio y } \\
\text { Mandato S.A. } \\
\text { Maquinarias y }\end{array}$ & Comercio & Guayas & $\mathrm{x}$ & $\mathrm{x}$ & $\mathrm{x}$ & $\mathrm{x}$ & $\mathrm{x}$ & $\mathrm{x}$ \\
\hline 88 & $\begin{array}{l}\text { Vehículos S. A. } \\
\text { MAVESA }\end{array}$ & Comercio & Guayas & $\mathrm{x}$ & $\mathrm{x}$ & $\mathrm{x}$ & $\mathrm{x}$ & $\mathrm{x}$ & $\mathrm{x}$ \\
\hline 89 & $\begin{array}{l}\text { La Ganga R.C.A. } \\
\text { S.A. }\end{array}$ & Comercio & Guayas & $\mathrm{x}$ & $\mathrm{x}$ & $\mathrm{x}$ & $\mathrm{x}$ & $\mathrm{x}$ & $\mathrm{x}$ \\
\hline
\end{tabular}




\begin{tabular}{|c|c|c|c|c|c|c|c|c|c|}
\hline No & Nombre & Sector Económico & Provincia & Historia & Misión & Visión & $\begin{array}{l}\text { Estrategia } \\
\text { competitiva }\end{array}$ & $\begin{array}{l}\text { Diferenciación } \\
\text { en el mercado }\end{array}$ & $\begin{array}{c}\text { Atributos } \\
\text { de la } \\
\text { Marca } \\
\end{array}$ \\
\hline 90 & $\begin{array}{l}\text { Productos Avon } \\
\text { (Ecuador) S.A. }\end{array}$ & Comercio & Pichincha & $\mathrm{x}$ & $\mathrm{x}$ & $\mathrm{x}$ & $\mathrm{x}$ & $\mathrm{x}$ & $\mathrm{x}$ \\
\hline 91 & $\begin{array}{l}\text { Automotores y } \\
\text { Anexos S.A. } \\
\text { (A.Y.A.S.A.) }\end{array}$ & Comercio & Pichincha & $\mathrm{x}$ & $\mathrm{x}$ & $\mathrm{x}$ & $\mathrm{x}$ & $\mathrm{x}$ & $\mathrm{x}$ \\
\hline 92 & $\begin{array}{l}\text { Automotores } \\
\text { Continental S.A. }\end{array}$ & Comercio & Pichincha & $\mathrm{x}$ & $\mathrm{x}$ & $\mathrm{x}$ & $\mathrm{x}$ & $\mathrm{x}$ & $\mathrm{x}$ \\
\hline 93 & Masgas S.A. & Comercio & Pichincha & $\mathrm{x}$ & $\mathrm{x}$ & $\mathrm{x}$ & $\mathrm{x}$ & $\mathrm{x}$ & $\mathrm{x}$ \\
\hline 94 & $\begin{array}{l}\text { Int Food Services } \\
\text { Corp }\end{array}$ & $\begin{array}{l}\text { Alojamiento y } \\
\text { servicios de } \\
\text { comida }\end{array}$ & Pichincha & $\mathrm{x}$ & $\mathrm{x}$ & $\mathrm{x}$ & $\mathrm{x}$ & $\mathrm{x}$ & $\mathrm{x}$ \\
\hline 95 & $\begin{array}{l}\text { Contecon } \\
\text { Guayaquil S.A. }\end{array}$ & Transporte & Guayas & $\mathrm{x}$ & $\mathrm{x}$ & $\mathrm{x}$ & $\mathrm{x}$ & $\mathrm{x}$ & $\mathrm{x}$ \\
\hline 96 & $\begin{array}{l}\text { Aerolane Líneas } \\
\text { Aéreas Nacionales } \\
\text { del Ecuador S.A. }\end{array}$ & Transporte & Pichincha & $\mathrm{x}$ & $\mathrm{x}$ & $\mathrm{X}$ & $\mathrm{x}$ & $\mathrm{x}$ & $\mathrm{x}$ \\
\hline 97 & $\begin{array}{l}\text { Consorcio } \\
\text { Ecuatoriano de } \\
\text { Telecomunicaciones } \\
\text { S.A. }\end{array}$ & $\begin{array}{l}\text { Correo y } \\
\text { comunicaciones }\end{array}$ & Guayas & $\mathrm{x}$ & $\mathrm{x}$ & $\mathrm{x}$ & $\mathrm{x}$ & $\mathrm{x}$ & $\mathrm{x}$ \\
\hline & CONECEL & & & & & & & & \\
\hline 98 & Otecel S.A. & $\begin{array}{l}\text { Correo y } \\
\text { comunicaciones }\end{array}$ & Pichincha & $\mathrm{x}$ & $\mathrm{x}$ & $\mathrm{x}$ & $\mathrm{x}$ & $\mathrm{x}$ & $\mathrm{x}$ \\
\hline 99 & $\begin{array}{l}\text { Directv Ecuador } \\
\text { Cía. Ltda. }\end{array}$ & $\begin{array}{l}\text { Correo y } \\
\text { comunicaciones }\end{array}$ & Pichincha & $\mathrm{x}$ & $\mathrm{x}$ & $\mathrm{x}$ & $\mathrm{x}$ & $\mathrm{x}$ & $\mathrm{x}$ \\
\hline 100 & $\begin{array}{l}\text { Saludsa Sistema de } \\
\text { Medicina pre- } \\
\text { pagada del Ecuador } \\
\text { S.A. }\end{array}$ & $\begin{array}{l}\text { Enseñanza y } \\
\text { servicios de } \\
\text { salud }\end{array}$ & Pichincha & $\mathrm{X}$ & $\mathrm{X}$ & $\mathrm{X}$ & $\mathrm{X}$ & $\mathrm{X}$ & $\mathrm{X}$ \\
\hline
\end{tabular}

Nota: Esta tabla es el resultado de una tabulación de resultados de una investigación de campo con base a: llamadas telefónicas a las empresas, investigación en revistas especializadas en rankings empresariales como:

Vistazo y Ekos, varios números.

\section{Conclusiones}

En promedio las empresas analizadas en el estudio crecieron en términos reales $12,3 \%$, cuando la economía en el mismo período creció al $3 \%$ promedio anual y en los respectivos sectores el 3,3\%, permitiendo que los sectores económicos en los que participaron crezcan más que el promedio de la economía. Aunque hay sectores económicos y empresas mejor clasificadas 
por ingresos que tuvieron en conjunto decrecimientos en los sectores manufactureros, de comercio y de suministro de agua, existe un impacto significativo más bien positivo de la muestra.

La participación de estas firmas en el total de los ingresos de los sectores de la economía con los que se corresponden equivale al $44 \%$ y en el total de la economía es el $33 \%$ de los ingresos. Por ejemplo, si la economía ecuatoriana presenta una renta total de 100 mil millones de dólares, estas firmas tienen ingresos de aproximadamente 33 mil millones de dólares en un año.

En materia de empleo, al comenzar el período de 2010, las 100 firmas daban lugar a 116.451 plazas de trabajo y al finalizar el año 2016, 158.641 plazas, con un incremento en 7 años de 42.190 plazas de trabajo. Comparado con el empleo pleno, diferente del empleo precario e informal proporcionado por las pequeñas empresas y las microempresas, el empleo total creció de 2`875.533 en plazas a 3’243.293 en el año 2016; por tanto, la participación pasó del 4,05\% al $4,88 \%$ como impacto en este tipo de empleo pleno: formal, de buena calidad y sujeto a todas las formalidades demandadas por la normativa laboral de Ecuador.

En materia de inversión, la formación bruta de capital en valores constantes por parte del sector privado en su totalidad fue de 67.476 millones de dólares. La contribución de las cien firmas a la expansión de esta inversión fue del 20,9\%, es decir de 14.124 millones de dólares. La alta productividad de estas firmas, impactó positivamente en los niveles de formación bruta de capital, al menos en una quinta parte de toda la capitalización. En general, altos niveles de capitalización, permiten mejorar la productividad y por tanto el ingreso y bienestar para todas aquellas personas que han sido parte de las historias de éxito empresarial, en estas cien firmas, que son las líderes en las respectivas ramas de actividad en las que operan.

Si bien no fue posible efectuar un análisis microeconómico de los precios de los bienes y servicios ofertados por estas cien firmas, se analizó la evolución del deflactor del PIB sectorial, es decir del índice de precios de las once ramas de actividad del presente estudio. Las conclusiones son las siguientes: en la manufactura, construcción, enseñanza, alojamiento y servicios de comida, los precios subieron algo más que la inflación promedio de toda la economía que fue del 2,1\% anual. En todos los demás sectores, la inflación o evolución de los precios fue menor. Algunos sectores como el petrolero y el de la acuicultura sufrieron una caída de los precios y por tanto de la inflación, debido al comportamiento de los precios internacionales del mercado.

El éxito de estas firmas no solo depende de sus ventas, sino del resultado responsable ante las crecientes expectativas de accionistas, inversionistas, empleados, consumidores y de la sociedad en general, stakeholder. (FORBES, 2014)

$\mathrm{Al}$ igual que las marcas famosas en el mundo empresarial, se puede anotar que las cien firmas ecuatorianas más importantes han desarrollado sus propias marcas asociadas con la historia de la propia firma, lo que constituye una ventaja si estas son favorables o interesantes. Marcas como La Favorita o las creadas por Pronaca, indudablemente están relacionadas con una historia de éxito empresarial. (Kotler, 1999) 
Las firmas aprenden a ser flexibles para adaptarse a los cambios en la competencia y el entorno, cultivando unas cuantas competencias esenciales para ser las líderes de una industria aventajando a sus rivales. Muchas de las cien firmas han superado muchas barreras competitivas debido a la liberalización y globalización y lo han logrado con mucha agilidad. (Porter, 1999)

Para entender el concepto de función empresarial, nos hemos remitido a lo que manifiesta Jesús Huerta de Soto, para quien la función empresarial coincide con la acción humana misma, en la visión misesiana o praxeológica de este concepto, es decir todo individuo actúa buscando los fines más valiosos utilizando los medios más útiles y afrontando a cada momento el fenómeno económico de la escasez, en el que prueba su perspicacia empresarial. Sujeta su acción a un plan (estrategia) que, si la ejecuta en un tiempo empresarial, como un evento único para cada firma, sujeto siempre a una incertidumbre inerradicable, finalmente alcanza utilidades o pérdidas puras. Este concepto se aplicaría a cualquier empresa en un sentido amplio. (Huerta de Soto, 1992)

\section{Bibliografía}

Banco Central del Ecuador. (octubre de 2017). Estadísticas Económicas. Obtenido de Publicaciones generales: https://www.bce.fin.ec/index.php/component/k2/item/776

Ferrel, O. C. (2010). Introducción a los Negocios. México: MacGraw Hill.

FORBES, R. d. (12 de 08 de 2014). FORBES. Obtenido de https://www.forbes.com.mx/comoasegurar-el-exito-de-tu-empresa/

Huerta de Soto, J. (1992). Socialismo, cálculo económico y función empresarial. Madrid: Unión Editorial S.A.

INEC, I. N. (06 de 2018). Instituto Nacional de Estadísiticas y Censos INEC. Obtenido de INEC Recuperado de http://www.ecuadorencifras.gob.ec/documentos/webinec/EMPLEO/2018/Junio-2018/062018_Mercado\%20Laboral.pdf

Koontz, H. (2012). Administración. México: MacGraw Hill.

Kotler, P. (1999). El Marketing según Kotler. Buenos Aires: Paidós.

Mankiw, N. G. (2012). Principios de Economía. México: Cengage Learning Editores.

Mises, L. V. (2005). La Accion Humana. Madrid: Unión Editorial.

Porter, M. E. (1999). Ser Competitivo. Bilbao: Deusto.

Pride, W. M. (2017). Introducción a los negocios. México: Cengage Learning.

Ravier, L. (2016). Historia Económica de la Empresarialidad, versión Kindle. Madrid, España: Unión Editorial, s.a.

Superintendencia de Compañías, V. y. (28 de 09 de 2018). Superintendencia de Compañías, Valores y Seguros. Obtenido de https://appscvs.supercias.gob.ec/rankingCias/

Vistazo. (2016-2017). 500 Mayores Empresas del Ecuador. Vistazo, p.140-144 y 198-206.

Vistazo. (2016). 500 Mayores Empresas del Ecuador. Vistazo, p.155-344.

Vistazo. (2015). 500 Mayores Empresas del Ecuador. Vistazo, p.189-314.

Vistazo. (2014). 500 Mayores Empresas del Ecuador. Vistazo, p.91-254.

Vistazo. (2013). Las 100 Empresas y Lideres con mayor reputación en el Ecuador Vistazo, p.107-198. 